\title{
reviews
}

columns

\section{Integrated Management of Depression in the Elderly}

Edited by Carolyn A. Chew-Graham, Robert Baldwin \& Alistair Burns Cambridge University Press, 2008, f29.99 pb, $198 \mathrm{pp}$.

ISBN 978-0-521-68980-9

This book offers a rare blend of academic studies and clinical experience. As depression in the elderly is undertreated and certainly underdetected, this publication becomes an essential resource to lessen the detrimental impact of depression on the outcome of care for physical and psychiatric disorders in this patient group. The authors manage to make us think of depression as an illness that could be successfully treated in primary care. This could be achieved in an integrated model that would bring specialist knowledge to primary care as well as ensuring that individuals with complex cases of depression will have easy access to secondary care expertise.

The opening chapter gives a wellstudied and summarised overview of epidemiology, aetiology and diagnosis of depression in late-life with a beautifully described account of the same issues in ethnic minority groups. There is more about the stepped care approach to the management of depression in the second chapter, supported in the third chapter with a number of case studies that practically demonstrate the experiences of some practitioners with this approach The authors continue to use clinical cases to illustrate the use of an 'integrated approach' in the management of individuals with depression with psychiatric and physical comorbidity. Chapter six is one of the best chapters in the book, giving a wide-ranging but easily digestible account of practical available resources for practitioners.

The book gives a comprehensive and enjoyable summary about depression screening tools for the elderly. The authors provide a road map to find out many resources for the treatment of depression: official sites, voluntary organisations, patient education materials and self-help materials. There is a very brief description of some legal concepts, but that turns to be diluted and insufficient. The management of depression in many countries around the world is outlined in an appendix.

I believe that the authors have enriched our resources by producing this extremely useful book that should be made easily available to all primary care practitioners and psychiatrists who are interested in making an impact on the treatment of depression in the elderly.

George Tadros Consultant in Old Age Psychiatry, Birmingham and Solihull Mental Health Foundation Trust, and Professor of Mental Health and Ageing, Staffordshire University, email: george.tadros@ bsmht.nhs.uk

doi: 10.1192/pb.bp.108.021469

\section{Depression (Oxford Psychiatry Library Series)}

Raymond W. Lam \& Hiram Mok Oxford University Press, 2008, f5.99 pb, $120 \mathrm{pp}$. ISBN 978-0-19-921988-9

This book is intended as a practical bedside reference. The authors make clear from the outset that they have simplified a complex diagnosis to give an overview of the topic and its management.
The opening chapter gives several vignettes of how depression might present in clinical practice and the following chapters outline its epidemiology, pathogenesis, clinical features and diagnosis. In the remaining chapters, the authors focus on depression management, various treatment modalities and treatment in special groups (the elderly, pregnant or breastfeeding women and adolescents). A useful collection of rating scales, both patient- and clinicianadministered, is included in the appendix.

The book is well laid out and easy to read. A large amount of detailed information has been condensed into this volume, with illustrations included in the chapter on pathogenesis. Illustrations are quite detailed, but a small font size makes them difficult to read. They are, however, well referenced, so that an interested reader can easily locate additional information.

Each chapter begins with a few key points and there are several tables making for easy reference. Those on prescribing are not as detailed as in the Maudsley prescribing guidelines but they are nevertheless useful and the book itself is much easier to carry around. It does cover other therapies, including the psychotherapies and somatic treatments, in good detail. As it is a Canadian publication, the DSMIV is the favoured diagnostic system and some of the treatments are not widely used in the UK, but most of the information is internationally relevant. I have already found it useful in my out-patient clinic.

Floriana Coccia Birmingham and Solihull Mental Health NHS FoundationTrust, Barberry Centre, 25 Vincent Drive, Birmingham B15 2FG, email: floriana. coccia@bsmhft.nhs.uk

doi: 10.1192/pb.bp.108.021519 